Makrufa Sh. Hajirahimova

DOI: $10.25045 /$ jpit.v08.i1.08

Institute of Information Technology of ANAS, Baku, Azerbaijan

makrufa@science.az

\title{
THE BIG DATA ERA IN HEALTHCARE: PROMISES AND CHALLENGES
}

Currently, Big data has become one of the driving forces of the development of information society. Big-data have the potential to transform health care. In this article, the essence of big data is described, the sources, possibilities of big data are interpreted in healthcare. Some created problems of big data in healthcare are also analyzed. The security issues of personal medical information is reviewed, recommendations related to the development of the healthcare sector are given.

Keywords: big data, healthcare, e-medicine, genome, biomedicine, telemedicine, electronic health records, personal medical data.

\section{Introduction}

Modern information technology has significantly changed our personal lives and our thinking. Real (physical) processes in the world have moved to online environment: acquisition of goods and services, communication, interaction between the employer and government agencies, and others. This situation has been caused by many factors: 1) penetration of the Internet into daily life; 2) emergence of e-commerce; 3) collection of information about the behavior of individuals on the Internet (establishment and development of estimated retrieval services, the core of which is a business model); 4) formation of social networks that aggregate the data not only on individuals, but also their relationships; 5) spread of smartphones and tablets ensuring the exchange of information at any time, and to be constantly online everywhere, to follow the route of the users' movement. As a result, a major part of human activity is moving to the Internet and any action of an individual leaves some tracks from so that the total of which causes the rapid increase in digital information. Suchan "explosion" of information in the world has become a serious problem called Big data. This problem, which presents in all areas of activity, is also common for the health sector [1-3]. Thus, as a result of the digitalization of health care from the traditional (paper) form, large amounts of different types of data are collected in Clinical Decision Support Systems (CDSS) of clinics, and this process is dynamically continuing. This data collection (patients' electronic medical records, medicines, electronic registry systems, etc.) differs from each other in volume, velocity and diversity $[4,5]$.

Big data in medicine is traditionally generated by means of the Internet, smartphones, social and mass media or, as mentioned above, by information systems of hospitals and clinics. How to save a large amount of information, how to manage it, or how to get useful information from it is a very serious problem. Since health data requires real-time processing for the diagnosis. The problem is the failure of processing of continuously generated data from different sources and in different formats (structured and unstructured) through traditional methods and tools [6]. Therefore, there is a need for more advanced technologies. The objective of the study case is to get readers and researchers familiar with the opportunities and challenges of Big data technologies in the health system, and to prove it to be a key condition and component in operational decisions motivated by the experts (doctors).

\section{Big data paradigm}

Web, social networks, mobile devices, transactions made through credit cards, business information systems, cameras, electronic health cards, and so on have led to an increase in the digital data stream, and as a result, the information abundance has emerged and the world is literally filled with information [6]. Since the beginning of the twenty-first century, digital data has grown in geometric progression each year. This is also confirmed by reports from companies such as International Data Corporation (IDC), Gartner and others, which are specialized in 
information technology [7-9]. The volume of information in the world in 2013 reached 4.4 zettabytes, only less than $2 \%$ of which was non-digital information. According to IDC's forecasts, the amount of digital information is growing by $40 \%$ per year. The amount of digital information in the world will reach 44 zettabytes by 2020 [7]. In addition, most data is forecasted to be generated not by the people, but due to the interaction of different devices, such as RadioFrequency Identification (RFID), Global Positioning System (GPS) between the years of 20122020. In the interaction of different types of devices via the Internet, based on the concept of the "Internet of Things", which is considered the next phase of the Internet development, the machines become not only the single manufacturer of data, but also its customers. As a result, Big data phenomenon emerged in the new era of data processing, storage and use $[2,3,6]$.

Is Big data really capable to improve the healthcare system, the option of appropriate treatment methods and to reduce other health problems? First, basic health attributes of Big data should be reviewed. In general, the era of Big data is based on the stages of the evolution of information technology: 1) data processing in the 1960s; 2) information systems in the 19701980s; 3) decision support models in the 1990s; 4) data warehouses in the 2000s; 5) establishment of the era of Big data since 2010 [10]. The term of "Big data" refers to a set of high-speed and large-scaled (from 1 terabyte up to zettabytes) data of a complex structure and various types. There are different approaches to the perception of this phenomenon offered by the interested parties as science, industry and business stakeholders. Regardless of the application areas, the main attributes of Big data are Volume, Velocity and Variety. These attributes were first offered by Gartner and recognized as a 3V model [8]. In McKinsey Global Institute report, the term is defined as data set, which is beyond the capabilities of a typical data storage, management, search and analysis database [9]. This model reflects the basic concept of Big data technologies. In terms of the strategy and management, professionals emphasize the need to improve this model, and consider measuring Big data by its characteristics as veracity and value $[11,12]$. In other words, data is so large that it is difficult to be handled, whereas extracting useful information from it is more difficult. Since, in this case, available technologies are inefficient.

The concept of Big data is not just a matter of size of the data, but a process of creating the value, and it is synonymous to the notions as "business intelligence", "business analytics", and "data mining" [10]. At present, open-source Hadoop, commercial Cassandra, Cloudera, Hortonworks, MapR and other technological platforms have been developed to solve the problem of big data.

Big data, currently, has covered all the aspects of human life, as well as biological and medical ones [13]. In recent years, in connection with the development of "omics" (genomics, metabolomics etc.) technologies in molecular biology, very large volumes of data have been generated. At the same time, the digitization of medical records has led to the exponential growth of data. Data analysis is creating great opportunities for doctors, epidemiologists and the policy makers in health care sector. [14] shows that Big data is not only a reality for biomedicine experts, but also an effective tool for the perception and search of new knowledge. Access to the data generated by Omics technologies has led to revolutionary changes in the field of medical biology, bioinformatics and other fields of science.

There is no unequivocally accepted definition of the term Big data in Medical Subject Headings. Therefore, the basic requirement is a common understanding of the term [15].

\section{The trends forming Big data potential}

The modern information society is characterized not only by the increase in the volume of information, but also by the increase of its role of information in various spheres of activity. Information is gaining the status of a new production factor called "new oil" and becoming the driving force of information society. Indeed, the traditional oil was a key resource in the industrial society. Experts think that the data acquisition, integration and analysis are no longer the expenditure for business, but the key to efficiency and income. 
Big data analysis and the acquisition of knowledge and useful information from it has created great opportunities for the revolutionary changes in management and business enterprises, obtaining high profits, development and implementation of scientific ideas in many areas, new scientific discoveries, and decisions made in organizations, national security, health care and so on $[6,16]$. Big data's potential is perceived by the state bodies, science, and business community. The following facts are proving it:

- Big data is regarded as a strategic resource as oil by governmental structures, scientific community, and business circles of the US and other Western countries.

- International conferences, seminars, forums on various aspects of Big data are held, and special editions of scientific and popular magazines are devoted to this subject. Since 2014, new academic journals titled "Big Data" have been published.

- Fundamental studies are being carried out on Big data collection and processing, storage, architecture, analytics, security, visualization, and so on in areas leading scientific centers of the world [3].

- Since 2013, a number of leading universities have taught the discipline "Data science" at bachelor, master and doctoral courses [6].

It is no coincidence that the IDC, McKinsey Global Institute, Gartner and others consider Big data as the key technological field of science and driving force of the ICT [2-4].

\section{Big data in healthcare - sources, opportunities and challenges}

As a rapidly rising segment of the digital world, healthcare itself reflects its large part. It can also be seen in the statistics of IDC's recent report [7]. As seen in the report, the volume of data generated in the health sector has been steadily increasing during last decade. The size of the data in the field of healthcare is expected to be 25000petabytes by 2020, while in 2012 it was 500petabytes. The reason for the increase is the emergence of new technologies; the prevalence of data collection devices, sensors, mobile medical applications; the fall in the cost of collection of genetic data; the increase in digital communications with the patients; acquiring more medical knowledge, and etc. [5].

Big data - sources in health. In general, IT applications in health are divided into two categories [4]:

- Clinical Systems. There are many systems in this category: Picture Archiving and Communications Systems (PACS), hospital information systems (HIS), Electronic Health Records (EHR), radiology information systems (RIS), Enterprise Content management (ECM), laboratory information System (LIS) and others.

EHR patient's medical information, including an electronic version of its demographic data, medical records, medications, vital, vital signs, passed a medical history, immune, laboratory, radiology and other reports reflect data.

- Administration Systems, text processing tools, spreadsheets, presentations, and even accounting software and so on.

Indeed, data is rapidly increasing in health care. In [17], the main sources of the rise are indicated as electronic health cards, biotechnology and academic research. This data analysis, in turn, has great potential for health and is significantly valuable.

Various types of data are generated by health systems, so that they can be divided into the categories as structured, unstructured, and partially structured:

- $\quad$ Structured electronic health records (EHR);

- Unstructured clinical records;

- Medical Imaging Data;

- Genetic data;

- Other data (Epidemiological and behavioral).

This classification suggests that data collected in health, as a rule, is characterized by $5 \mathrm{~V}$ [3]: 
- Volume: the patients' electronic medical records, medicines, the data generated and transmitted through electronic registry systems, medical electronic devices and so on. As stated in [4] being the rapidly rising segment of the digital world, healthcare includes its $30 \%$ portion.

- Variety: structured EHR, unstructured clinical records, other unstructured data such as video, audio and text contents and so on. $60-80 \%$ of health data includes unstructured data containing images, videos, and e-mail records [5, 18].

The emergence of digital medical facilities (especially in the fields of radiology, cardiology, oftomologiya etc.) since the mid-1980shas radically changed the face of medical visualization.Transmitting obtained digital image data to PACS systems, they are electronically processed in computers and stored along with demographic and vital data to study the patients better. As a result, large volumes of data are collected.

- Velocity: new data is collected in stream with high rate, and in some medical situations, data becomes a matter of life and death. Real-time data analysis (traumas monitoring, cardiac monitors, anesthesia, etc.), comparisons and decisions require high speed. It also includes daily diabetic measurements, blood pressure measurement, ECG (Electrocardiography).

- Veracity - data quality: in terms of health care it requires special attention for two reasons: 1) it is a matter of life or death, so that it depends on the order of information; 2) quality of health data, especially unstructured data (e.g., hand-written recipes) are often inexact. Healthcare patients, hospitals, payment amount, payers and so on are the main parameters for data authenticity. Other unique issues for data authenticity include diagnoses, prescriptions, treatments, and procedures. In other words, improvement of medical services, reducing recurrence of errors, accuracy of diagnosis, efficiency of medicines, reducing the cost depend on the quality of data.

- Value:

- real-time decision support;

- precise diagnosis;

- cost reduction;

- minimization of the risk of disease;

- use of data in research and so on.

Biomedical data. High performance biotechnology measurement technologies were developed in the 21 st century, and the studies on biomedical technologies are also conducted in the era of Big data. With the development of smart, efficient and accurate analytical models, and theoretical systems in biomedicine, an important control mechanism can be discovered at the background of complex biological events. It does not just provide future development of biomedicine, but also can be recognized for important strategic industrial development associated with the important applications as the national security and medical service, production of new medicines [19-21].

The Human Genome Project (HGP) and the subsequent development of long-term technological development have led to the broad applications of big data. Implementing specialized analysis of large-scale data generated by Genome Sequencing in accordance with various requirements and combining it with a clinical genetic diagnosis, valuable information can be given for the early diagnosis and individualized of disease treatment. One Genome Sequencing can generate 100-600GB of raw data. Shenzhen National Genome Bank of China stores total 1.3 million samples, including 1.15 million reviewable human samples and 150000 samples of animals, plants and micro-organisms.

We can predict in advance that, with the development of biomedical technologies, genome de-coding will be faster and more comfortable, so that large scale biomedical information will continuously be increased. At the same time, the data generated from the clinical medical aid and 
medical research is rapidly growing. For example, the US University of Pittsburgh Medical Center (UPMC) stores 2 TB data. Practice Fusion American manages electronic health records of approximately 200000 patients. Explorys, an American company, provides platforms to arrange clinical data, operational and technical data, and even financial data.

Telemedicine. Telemedicine, first of all, is high-quality technology, which provides the first aid and control to the patient. Telemedicine is one of the technologies of the twentieth century using a wide range of telecommunications and information technologies and providing medical services. It enables exchanging health data between doctor and patient to achieve the best results.

Big data is seriously changing the telemedicine. At present, a large number of devices that monitor the health of patients are constantly generating data, which is analyzed and collected in real-time mode. This analysis may detect the model of the disease and warn a patient and doctor about the first signs of abnormalities (especially in chronic diseases). A joint analysis of information from various sources about the patients can provide a better service. The larger the database, the more opportunities can be created for researches, new jobs and improved diagnostic methods, and for the prevention and treatment of random and widespread diseases.

The main advantage of telemedicine is that physician can observe the patient in real-time based on the data provided by means of smart devices without going to the hospital; he can also monitor the chronic disease, and define the treatment and the dose of the medication more precisely. Another aspect is the reduction of the costs. Telemedicine has now been widespread and integrated with daily activities of hospitals, private physicians and health agencies $[22,23]$. The key trends of telemedicine are: online and offline tele-consultations, surgical operations broadcast, bio-monitoring, and telemedicine at home.

\section{Big Data opportunities for health}

Big data analytics enables revolutionary changes in the healthcare system. Improving the efficiency of operations can facilitate to predict the epidemic diseases, optimize the costs in health sector, and improve the quality of the monitoring of clinical trials $[1-3,6,10,24]$.

The overall objectives of Big data in healthcare are:

- to ensure timely and appropriate intervention using large amounts of health data of the patients;

- to ensure individualized care for patients;

- to contribute to potential benefit to all components of the health care system (provider, payer, patient and management).

Big data can play an important role in detecting causes and consequences of disease signs of the patients, in predicting the rate of danger or re-occurrence of the disease, and also in providing the first aid. Big data in medicine can be used for diagnosing the disease, prognosis of disease prevention, managing individual health and disease, and for the creation of individual programs.

Big data technologies can also play an important role in the medical activities. Big data technologies in this field enables us to provide an individual approach to the treatment, in which the device using Big data technologies act as a personal assistant of the doctor. Data analysis based on advanced studies, experience in the treatment of an appropriate disease, and the clinical study of treatment drugs of the individual patient's, and individual treatment plan that takes into account the characteristics of an organism can be developed. Many companies are actively working in this field. For example, IBM is cooperating with many organizations for the development of the system that provides the analysis of human genes and defines the proper treatment of the patient as soon as possible. These systems collect information about genes, study the patient's reaction to the appointed treatment and offer alternatives, taking into account the nature of human DNA [24, 25]. 


\section{Big data challenges in health}

In addition to wide range of opportunities of the rapidly growing data in healthcare, it also creates a number of problems. These problems can be divided into three groups: technical and technological, juridical and scientific challenges [2, 3].

Technological challenges include software for Big data platforms in healthcare systems, their integration, user interfaces and so on.

In health care system, especially in e-medicine, the vast majority of the data is the personal data of the patients. In this respect, introduction of Big data analysis arises protection issues of citizens personal lives in the digital environment. The main problem here is related to the inconsistencies and contradictions in legislation appeared in the nature of the Big data analysis technologies and the personal data. The limitations on the amount of processed or re-used personal data to achieve the purposes specified in advance are contrary to the philosophy of Big data and they strip the capabilities of this technology $[26,27]$. We will review this problem in the next section in details.

European Union introduces the main problems of Big data technologies in the health sector as follows: data confidentiality, security, authenticity, relevance and control [4, 28].

Patients are concerning about their health data, in particular, about the acquisition of genetic data. Data availability and privacy risks are associated with each other. The reforms in the legislation are required with the Open Data policy of the European Commission to eliminate this threat.

As in all areas, serious problems emerge in the field storage, processing and analysis of large-scale data [28-30]:

- producing knowledge from the heterogeneous sources;

- $\quad$ using patient/data correlations in papers;

- understanding the unstructured clinical records in the proper context;

- effective processing of large volumes of data containing medical images and producing potentially important information and biomarkers;

- genetic data analysis is a matter of computing and creates additional difficulties if connected to standard clinical data;

- collecting the data about the patients' behavior through the sensors and social interactions.

More advanced mining and analysis techniques, visualization techniques and so on are required for the problem solution.

\section{Big Data applications in health}

After the adoption of the law on "Health Information Technology for Economic and Clinical Health" (HITECH) in the USA in 2009, EHR systems were introduced. The main advantage of EHR is the opportunity of providing the medical staff with complete and accurate health data. At present, most of the US hospitals (50\%) are using it.

The US is not the only country introducing the EHR. Canada has adopted "Canada Health Infoway" project as EHR for the acceleration of digital solutions for health care to improve the efficiency of the health service, medical care and medical services. The Government of Canada has allocated 2.15 billion Canadian dollars for the implementation of this project [31]. In Europe, 3 billion USD have been allocated the development of EHR technologies [28].

Medical Body Area Networks (MBAN) is included to another class of Big data applications in the field of healthcare. Wireless sensors MBAN enables continuous monitoring of the patient's condition by transmitting heartbeats, electrocardiogram, body temperature, respiratory frequency, chest sounds, blood pressure and so on. Furthermore, MBAN also provides real-time monitoring of the patient's health, infection control, and tracking the patient. Nevertheless, health care providers should have smart and high secure ICT infrastructure to realize real time analysis of such large MBAN data of hundreds of thousands of patients. 


\section{Personal health data}

Generally, specific provisions devoted to the problems of the automated processing of personal data first emerged in Europe and, later, were spread to the whole world. According to the reports for early 2012, the law on personal data has been adopted in 89 countries around the world. The main legal act in this area is the Convention on the protection of individuals of the Council of Europe in the automated processing of personal data dated January 28, 1981. According to the provisions of the Convention, European countries have adopted a national law on the regulation of personal data [28].

As a result of the digitalization of the health sector large volumes of personal data are included to the information systems. Studies show that in most health data is not protected enough and $7 \%$ of it is public and does not need to be protected, while most of it (93\%) is confidential and needs protection (for example, health records and stories). Statistic reports indicate that $57 \%$ of the patients' health data is partially protected, whereas $43 \%$ is adequately protected [7].

Medical and health data is most intensively exchanged data. The era of big data, the interaction between IT, computer science and researches is wider in the field of the healthcare. Today, the most pressing problems in medical institutions to be solved is the protection of confidential personal health data.

The personal information in the legislation is any data that directly or indirectly identifies the person [32]. Transmission of this documented information to a third party without the consent of its owner is prohibited by law. The owner of this secret health data is a patient or a legal representative with his/her consent. Personal data processing means any operation on them (its collection, classification, storage, update, modification, use, distribution, and destruction). Personal data processing at the medical institutions should be implemented in accordance with the rules ensuring the data confidentiality and protecting unauthorized interference.

Since the majority of data is the personal information, Big data technologies creates serious problems in terms of privacy. Therefore, this data should be kept private.

As noted in [12], the modern tracking technology and Big data analytics gives us the opportunity to track the people "from birth until death", which puts under question the integrity of his personal life. Analysis of the data of people without their consent is ethically and legally unacceptable.

\section{The future model of health care}

The followings are recommended for the future development model of the healthcare:

- Increasing the efficiency in all areas of the healthcare;

- Being more Collaborative/self-care);

- Drawing special attention to the control of public health.

The data will enable knowledge-based decisions, in which IT will be a key factor. To this end, the followings are recommended:

- to improve and expand the IT capabilities and rights within the framework of the working processes and practices;

- to choose appropriate analytical technologies and environment;

- to ensure more effective management of security;

- to adopt more powerful mobile strategies;

- cloud applications for healthcare based on coordination and team;

- to train Data Scientist to conduct research on health professionals.

\section{Conclusion}

We live in the world with full of very large scale data. Experts rightly call the 21st century - the era of Big data, which covers all aspects of human life, including healthcare. The increase in the volume and diversity of data shows that the era of Big data emerged for the healthcare sector. In addition, it has a great influence not only on the implementation and evaluation of information 
systems, but also on the development of researchers and professionals in the field of computer science. What medical experts should know in the field of computer science - what those who work in the field of Big data and analytics should know.

Transition to the electronic health cards from the traditional health recordings, and biomedicine and telemedicine facilities have exponentially increased the health data. As a result, Big data analytics provides opportunity to doctors and health experts great opportunities for decision making and conducting researches. Leading trends in healthcare sector will expand the volume of data and the dependence on them. In such environment, personal health data should be particularly protected. For more efficient use of data, there is a need for the professionals trained in medical biology and medical informatics and other areas.

\section{REFERENCES}

1. Zhang Sh.Big Data for Healthcare in China: a Review of the State-of-art // Cambridge Journal of China Studies, 2015, vol.10, no.1, pp.32-40.

2. Sebastian S.V., Haimann A., Mossialos E. Big Data and Health Care: Challenges and Opportunities for Coordinated Policy Development in the EU // Health Systems \& Reform, 2015, vol.1, no.4, pp.285-300.

3. Susan E.W.A review of big data in health care: challenges and opportunities // Open Access Bioinformatics, 2014, no.6, pp.13-18.

4. Whitepaper: Healthcare Disaster Recovery. www.bridgeheadsoftware.com

5. R. Wyber, etal.Big data in global health: improving health in low- and middle-income countries. www.who.int/bulletin/volumes/93/3/14-139022.pdf

6. Alguliyev R.M., Hajirahimova M.S. "Big Data" phenomenon: Challenges and Opportunities // Problems of Information Technologies, 2014, No2, pp.3-16.

7. The Digital Universe in driving data growth in healthcare. 2014. www.emc.com/collateral/analyst-reports/idc-the-digital-universe-in healthcare.pdf

8. Laney D. "3D Data Management: Controlling Data Volume, Velocity and Variety.” Technical report, META Group, Inc (now Gartner, Inc.), February 2001. http://blogs.gartner.com/

9. Big data: The next frontier for innovation, competition, and productivity. Analyst report, McKinsey Global Institute, May 2011. www.mckinsey.com/

10. Jee K., Kim G.H. Potentiality of Big Data in the Medical Sector: Focus on How to Reshape the Healthcare System // Healthcare Informatics Research, 2013, vol.19, no.2, pp.79-85.

11. Hajirahimova M.S. Big Data technologies and information security challenges // Problems of Information Technologies, 2016, No1, pp.49-56.

12. Imamverdiyev Y.N. Big prospects and problems of Big Data technology //Problems of Information Society, 2016, No1, pp.23-34.

13. Zhang Z. Big data and clinical research: focusing on the area of critical care medicine in mainland China // Quantitative Imaging in Medicine and Surgery, 2014, vol.4, no.5, pp.426-429.

14. Margolis R., Derr L., Dunn M. et al. The National Institutes of Health's Big Data to Knowledge (BD2K) initiative: capitalizing on biomedical big data // Journal of the American Medical Informatics Association, 2014, vol.21, no.6, pp. 957-958.

15. Baro E., Degoul S., Beuscart R., and Chazard E. Toward a Literature-Driven Definition of Big Data in Healthcare // BioMedResearch International, vol.2015, Article ID 639021, 9 pages.http://dx.doi.org/10.1155/2015/6390212014

16. Mayer-Shenberger V. Kukier K. Big data. A revolution that will change the way we live, work and think, M.: MIF, 2014, p.17.

17. Buchan I., Bishop Ch.A Unified Modelling Approach to Data-Intensive Healtcare. The fourth paradigm, 2009, pp.91-97.

18. http://medcitynews.com/2013/03/the-body-in-bytes-medical-images-as-a-source-ofhealthcare-big-data-infographic 
19. Toga A.W., Dinov I.D. Sharing big biomedical data // Journal of Big Data, 2015, vol.2, no.7, pp.1-12.

20. Trifonova O.P., Ilyin V.A., Kolker E.V., Lisitsa A.V. Big data in biology and medicine // Acta Naturae, 2013, Volume 5, No3 (18), pp.14-17.

21. Otero P., Hers W.H, Ganesh J. Big Data: Are Biomedical and Health Informatics Training Programs Ready? // Yearbook of Medical Informatics, 2014, pp.177-181. http://dx.doi.org/10.15265/IY-2014-0007

22. Coakley M., Crocetti G., Dressner P., Kellum W. Transforming Telemedicine Through Big Data Analytics. Cornell University Library, 2015.

23. What is Telemedicine? American Telemedicine Association. www.americantelemed.org/

24. Wullianallur R., Viju R.Big Data Analytics in Healthcare: Promise and Potential //Health Information Science and Systems, 2014, no.2, vol.3, pp.1-10.

25. Big data at the speed of business. 2015. www-01.ibm.com/software/data/bigdata/

26. Tene O., Polonetsky J. Privacy in the age of big data: A time for big decisions //Stanford Law Review Online, 2012. http://www.stanfordlawreview.org/online/privacy-paradox/big-data

27. Greenleaf G. Global Data Privacy Laws: 89 Countries, and Accelerating, Privacy Laws \& Business International Report, February 2012.

28. The Use of Big Data in Public Health Policy and Research, 2014. http://ec.europa.eu/research/health/pdf/public-health-research_en.pdf

29. Herland M., Khoshgoftaar T M., Wald R. A review of data mining using big data in health informatics // Journal of Big Data, 2014, vol.1, no.2, pp.1-35.

30. Alyass A., Turcotte M., Meyre D. From big data analysis to personalized medicine for all: challenges and opportunities //BMC Medical Genomics, 2015, vol.8, no.33, pp.1-12.

31. Finance, Government of Canada, Department of. "Budget 2016: Chapter 5 - An Inclusive and Fair Canada". www.budget.gc.ca. Retrieved2016-05-02.

32. The Law on Personal Data of the Republic of Azerbaijan, May 11, 2010, www.president.az 